\title{
Luminescence Decay Kinetics in GaN Studied by Frequency Domain Measurements
}

\author{
J. Mickevičius ${ }^{a}$, P. Vitta ${ }^{a}$, G. Tamulaitis $^{a}$, A. Žukauskas ${ }^{a}$, \\ M.S. $\mathrm{SHUR}^{b}$, J. ZHANG ${ }^{c}$, J. YANG ${ }^{c}$ AND R. GASKA \\ ${ }^{a}$ Institute of Materials Science and Applied Research, Vilnius University \\ Saulètekio al. 9-III, LT-10222, Vilnius, Lithuania \\ ${ }^{b}$ Department of ECE and CIE, Rensselaer Polytechnic Institute \\ Troy, NY 12180, USA \\ ${ }^{c}$ Sensor Electronic Technology, Inc. \\ 1195 Atlas Road, Columbia, SC 29209, USA
}

\begin{abstract}
Carrier dynamics in high-quality GaN epilayer was investigated at two extreme excitation levels. Carrier lifetime under high excitation conditions was estimated by using light-induced transient grating technique. Measurements at extremely low excitation power density were performed by using frequency-domain fluorescence lifetime technique. The study was performed in a wide temperature range from 8 to $300 \mathrm{~K}$. The results revealed the influence of donor-acceptor pair recombination and carrier trapping processes.
\end{abstract}

PACS numbers: 72.20.Jv, 78.55.Cr

\section{Introduction}

GaN and related alloys are widely investigated due to their possible applications in optoelectronic devices operating in blue and ultraviolet spectral regions. Extensive studies on nitrides indicate that the quality of III-nitride epilayers is still a major challenge, as the layers still contain large densities of defects. The quality of GaN epilayers is often characterized by the nonequilibrium carrier lifetime $[1,2]$, which is usually extracted from the time-resolved photoluminescence experiments. However, the reported time-resolved measurements were performed at relatively high excitation levels, when nonradiative and defect-related radiative recombination channels might have been partially or completely saturated and their effect on the carrier lifetime was reduced or eliminated. 
In this work, in addition to the conventional time-resolved measurements at high excitation level, we investigated the carrier dynamics in GaN epilayer at extremely low excitation power density. The studies at low excitation levels were performed using frequency-domain fluorescence lifetime (FDFL) measurements, while the light-induced transient grating (LITG) technique was used for measurements at high excitation level.

\section{Experimental}

The GaN epilayer used in this study was deposited on a high-quality AlN buffer layers grown on sapphire substrate using migration-enhanced metal-organic chemical vapor deposition (MEMOCVD ${ }^{\mathrm{TM}}$ ) [3]. The subsequent $10 \mu \mathrm{m}$ thick GaN layer was grown by conventional low-pressure metal-organic chemical vapor deposition. The sample was unintentionally $n$-type doped.

The LITG technique (see Ref. [4] for more details) was applied to estimate the carrier lifetime at room temperature at high carrier density, which is typical in operating light emitting devices.

To study the photoluminescence decay at extremely low carrier densities, FDFL technique [5] was employed. A $280 \mathrm{~nm}$ light emitting diode (LED) from Sensor Electronic Technology, Inc. was used as an excitation source in the FDFL experiments. The LED emission and, thus, the photoluminescence (PL) signal were modulated in the frequency range from $5 \mathrm{~Hz}$ to $200 \mathrm{MHz}$. The Fourier transform analysis was used to extract characteristic decay times. To reveal the competition of different recombination channels, the FDFL experiments were performed in a wide temperature range from 8 to $300 \mathrm{~K}$.

\section{Results and discussion}

The GaN sample under study was characterized at room temperature by using LITG technique. The excitation power density of $\sim 4 \mathrm{MW} / \mathrm{cm}^{2}$ used in these experiments was high enough to saturate trapping levels and defect-related recombination channels. The observed LITG decay could be described by a single exponent, and the estimated carrier lifetime was 2 ns. Such a long carrier lifetime indicates a high quality of the sample under study.

The measurements in frequency domain were performed at extremely low excitation power density of $\sim 0.5 \mathrm{~mW} / \mathrm{cm}^{2}$. Under that low excitation level, the PL spectrum of GaN epilayer contained two luminescence bands: a broad band centered around $2.2 \mathrm{eV}$ (yellow luminescence (YL) band) and a sharp peak at $3.490 \mathrm{eV}$ followed by phonon replicas (called near-band-edge emission, NBE, in the text below). The two spectral regions were analyzed separately in our FDFL experiments.

The typical phase shift curve of the NBE luminescence is shown in Fig. 1. It consists of the phase shift peak at $\sim 1 \mathrm{kHz}$ and a rising edge at high frequencies. Such phase shift curve can be adequately described by the superposition of two 


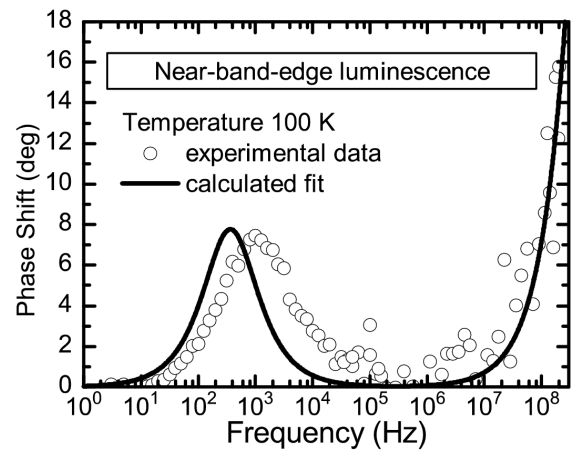

Fig. 1. Phase shift frequency dependence of the near-band-edge luminescence in FDFL experiments (points) at $100 \mathrm{~K}$ temperature. The solid line indicates the best fit using two-exponential decay.

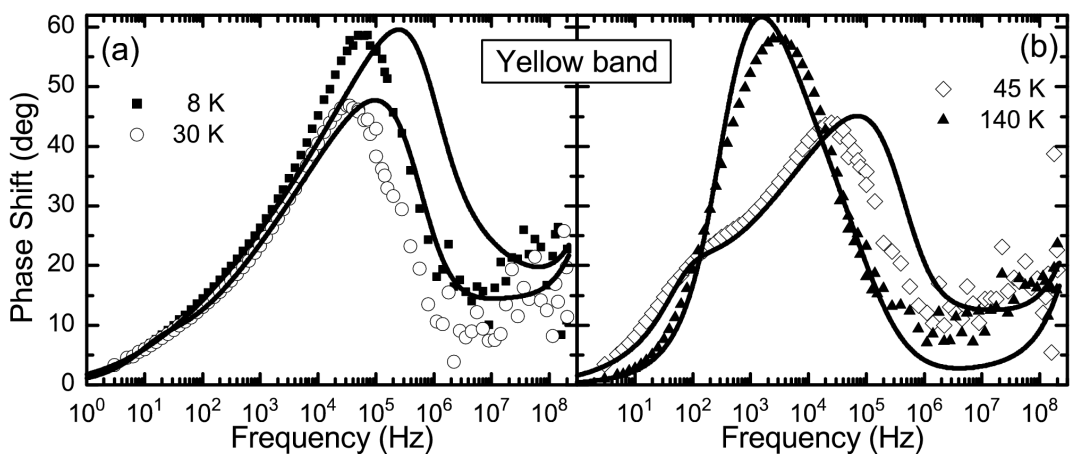

Fig. 2. Phase shift frequency dependences of the yellow band (points) in FDFL experiments at different temperatures (indicated). Solid lines show the calculated fit curves.

exponential decay components, as shown by solid line in Fig. 1. The characteristic times of the components are $500 \mu \mathrm{s}$ and 200 ps. Whereas Fig. 1 presents the data measured at $100 \mathrm{~K}$, the same spectral features were present in the entire temperature range used in this study.

The temperature evolution of the phase shift is more complicated for the yellow band (see Fig. 2). At the lowest temperatures, the observed phase shift increase in the range from $5 \mathrm{~Hz}$ to $\sim 40 \mathrm{kHz}$ was too slow to be described by an exponential decay. This phase shift can be explained by the recombination via the donor and acceptor states. Such donor-acceptor pair (DAP) recombination has been observed in GaN previously [6]. The recombination rate depends on the distance between donor and acceptor: the closer are the pairs, the faster is the recombination rate. Solid lines in Fig. 2a show the fits of the phase shift calculated using the DAP recombination model to the experimental data. Since a single DAP component could not properly describe the drop in the phase shift at high frequencies and its subsequent slight increase at even higher frequencies, an addi- 
tional exponential component was also included into the fitting function. Based on experimental data, it was assumed that the same component could participate both in NBE and in YL decay dynamics. A 200 ps decay time of the exponential component ensured the best fit.

With increase in temperature above $30 \mathrm{~K}$, one more additional decay component emerges. At $30 \mathrm{~K}$ it can be observed as a small phase shift peak at $\sim 10 \mathrm{~Hz}$ (see Fig. 2a), and its characteristic time was estimated to be $\sim 10 \mathrm{~ms}$. With further increase in temperature, the phase shift peak increases and its characteristic time decreases to $\sim 500 \mu$ s (see Fig. 2b). At temperatures of $\sim 100 \mathrm{~K}$, this decay component stops changing and stays the same throughout the rest of the temperature range. Such behavior might be related to the thermal ionization of a shallow donor. The depth of the donor level $\sim 15 \mathrm{meV}$ was estimated from the temperature dependence of the characteristic decay time.

The observed complex behavior of the luminescence decay dynamics in GaN epilayer can be explained by using the band model schematically shown in Fig. 3 . At low temperatures, the yellow band is caused by DAP transitions. With increase in temperature, the donor level is thermally ionized, and the band-to-acceptor (e-A) recombination gradually replaces the DAP component in the formation of the YL band. Some contribution from the DAPs, however, remains up to the room temperature.

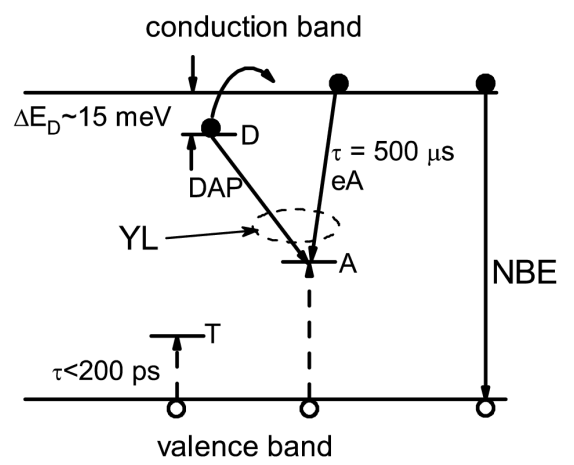

Fig. 3. Band model illustrating carrier dynamics in GaN. Donor, acceptor, and trap levels are indicated by $\mathrm{D}, \mathrm{A}$, and $\mathrm{T}$, respectively. Solid lines indicate radiative transitions; dashed lines correspond to nonradiative transitions.

The fast decay component at low temperatures might be attributed to the bound exciton recombination. This channel competes with the hole capture by the deep acceptors and, thus, contributes to the YL dynamics. Thermal ionization of bound excitons results in generation of free holes, which gives rise to the slower component in the NBE recombination. The decay of this weak component is due to the decay of the electron density caused by the e-A recombination with the characteristic time of $\sim 500 \mu \mathrm{s}$. This component, however, disappears at high 
temperatures due to hole trapping to levels deep enough not to be thermally ionized even at room temperature. Most probably, the trap density is low enough to be saturated at higher densities of nonequilibrium carriers. However, these traps are important at extremely low excitation levels and might affect the carrier dynamics at the late stages of carrier density decay after pulsed excitation.

\section{Conclusions}

In summary, our studies of carrier dynamics in GaN epilayer in a wide range of excitation intensities, including those corresponding to extremely low densities of nonequilibrium carriers enabled us to investigate the luminescence decay in a wide time window from sub-nanoseconds to milliseconds using the FDFL technique. The competition of different recombination channels responsible for yellow band in $\mathrm{GaN}$ and the role of hole traps was revealed.

\section{Acknowledgments}

The work at RPI was partly supported by the Office of Naval Research (Project Monitor Dr. Colin Wood).

\section{References}

[1] S. Juršėnas, N. Kurilčik, G. Kurilčik, S. Miasojedovas, A. Žukauskas, T. Suski, P. Perlin, M. Leszcynski, P. Prystawko, I. Grzegory, Appl. Phys. Lett. 85, 952 (2004).

[2] X.Y. Sun, R. Bommena, D. Burckel, A. Frauenglass, M.N. Fairchild, S.R.J. Brueck, G.A. Garrett, M. Wraback, S.D. Hersee, J. Appl. Phys. 95, 1450 (2004).

[3] R.S. Qhalid Fareed, R. Jain, R. Gaska, M.S. Shur, J. Wu, W. Walukiewicz, M. Asif Khan, Appl. Phys. Lett. 84, 1892 (2004).

[4] J. Mickevičius, M.S. Shur, R.S.Qhalid Fareed, J.P. Zhang, R. Gaska, G. Tamulaitis, Appl. Phys. Lett. 87, 241918 (2005).

[5] J.R. Lakowicz, Principles of Fluorescence Spectroscopy, Kluwer Academic, Plenum, New York 1999.

[6] R.Y. Korotkov, M.A. Reshchikov, B.M. Wessels, Physica B 325, 1 (2003). 